\title{
An Increase in Mitochondrial DNA Promotes Nuclear DNA Replication in Yeast
}

\author{
Heidi M. Blank, Chonghua Li, John E. Mueller, Lydia M. Bogomolnaya, Mary Bryk, Michael Polymenis*
}

Department of Biochemistry and Biophysics, Texas A\&M University, College Station, Texas, United States of America

\begin{abstract}
Coordination between cellular metabolism and DNA replication determines when cells initiate division. It has been assumed that metabolism only plays a permissive role in cell division. While blocking metabolism arrests cell division, it is not known whether an up-regulation of metabolic reactions accelerates cell cycle transitions. Here, we show that increasing the amount of mitochondrial DNA accelerates overall cell proliferation and promotes nuclear DNA replication, in a nutrientdependent manner. The Sir2p NAD -dependent de-acetylase antagonizes this mitochondrial role. We found that cells with increased mitochondrial DNA have reduced Sir $2 p$ levels bound at origins of DNA replication in the nucleus, accompanied with increased levels of $\mathrm{K} 9, \mathrm{~K} 14$-acetylated histone $\mathrm{H3}$ at those origins. Our results demonstrate an active role of mitochondrial processes in the control of cell division. They also suggest that cellular metabolism may impact on chromatin modifications to regulate the activity of origins of DNA replication.
\end{abstract}

Citation: Blank HM, Li C, Mueller JE, Bogomolnaya LM, Bryk M, et al. (2008) An Increase in Mitochondrial DNA Promotes Nuclear DNA Replication in Yeast. PLoS Genet 4(4): e1000047. doi:10.1371/journal.pgen.1000047

Editor: Orna Cohen-Fix, National Institute of Diabetes and Digestive and Kidney Diseases, United States of America

Received October 25, 2007; Accepted March 7, 2008; Published April 25, 2008

Copyright: (c) 2008 Blank et al. This is an open-access article distributed under the terms of the Creative Commons Attribution License, which permits unrestricted use, distribution, and reproduction in any medium, provided the original author and source are credited.

Funding: This work was supported by NIGMS grants to Michael Polymenis (R01-62377) and Mary Bryk (R01-70930).

Competing Interests: The authors have declared that no competing interests exist.

*E-mail: polymenis@tamu.edu

\section{Introduction}

Without cellular metabolism there is no cell division [1], but the key question is whether metabolism only allows for division to happen, or can it actively promote cell cycle progression. To determine if metabolism can actively promote cell division it is important to identify gain-of-function mutations in metabolic pathways that also accelerate cell proliferation. Such mutations have not been described in the yeast Saccharomyces cerevisiae. It is known, however, that yeast populations evolved in continuous chemostat cultures can proliferate faster than the parent population, and they have higher levels of tricarboxylic acid cycle (TCA) enzymes and mitochondrial (mt) DNA [2]. The mitochondrial genome is transmitted as a "nucleoid" DNA/protein complex. The number of mtDNA molecules per nucleoid varies, but there are usually more genome equivalents than nucleoids $[3,4]$. Abf2p is a conserved mtDNA maintenance protein $[5,6]$, which directly binds to, bends and compacts mtDNA $[7,8]$. Moderate over-expression of Abf2p by 2-3 fold elevates the amount of mtDNA by $50-150 \%$ [9]. The consequences of an increase in mtDNA in cell proliferation have not been explored.

Sir2p is an evolutionarily conserved $\mathrm{NAD}^{+}$-dependent deacetylase [10,11]. Loss of Sir $2 p$ leads to loss of transcriptional silencing, genome instability and a decrease in replicative life span. In yeast, silent chromatin is formed at three regions: the rDNA, the $H M L$ and $H M R$ mating type loci, and telomeres [12]. Sir2p is required for silencing at all of these regions, and it is the only Sir protein required for silencing at the rDNA [13-15]. Sir2p also appears to negatively impact on rDNA replication, because in $\operatorname{sir} 2 \Delta$ cells twice as many origins are activated within the rDNA array [16]. The inhibitory effects of Sir2p on DNA replication extend beyond rDNA. Loss of Sir2p suppresses replication defects of mutants that cannot assemble a pre-replicative complex of proteins (pre-RC) at origins of DNA replication in the Gl phase of the cell cycle [17]. These results may be linked to a general positive role of histone acetylation for origin activity [18]. Indeed, loss of the Rpd3p de-acetylase globally accelerated DNA replication, and targeted acetylation of a late origin advanced its activation [19], demonstrating a clear causal role of histone acetylation and activation of DNA replication. However, whether such chromatin modifications may serve as a link between cellular metabolism and initiation of DNA replication is not known.

In this report we show that an increase in mtDNA in cells overexpressing Abf2p, actively promotes initiation of cell division. Furthermore, we identify physical changes, such as Sir2p binding and histone acetylation, at an origin of DNA replication that result from an increase in mtDNA.

\section{Results/Discussion}

\section{Abf2p and Cell Proliferation in Chemostats}

We hypothesized that increasing the amount of mtDNA may mimic the situation of "evolved" yeast populations, which can proliferate faster than the parent population [2], allowing us to examine effects on cell division. We evaluated a strain $\left(3 \times A B F 2^{+}\right)$, which carries two additional copies of $A B F 2$, because in this strain the amount of mtDNA is increased [9]. The $3 x A B F 2^{+}$strain proliferated faster than the wild type strain in glucose-limiting $(0.08 \%$ glucose $)$ conditions (Figure 1A), mimicking "evolved" strains [2]. We next examined cell cycle progression in defined chemostat cultures under glucose (Glc) or nitrogen $(\mathrm{N})$ limitation at $0.2 \mathrm{~h}^{-1}$ dilution rate, $D$, comparing $A B F 2^{+}$to $3 x A B F 2^{+}$cells (Figure 1B). Under Glc-limitation, the fraction of $3 \times A B F 2^{+}$cells in G1 was reduced (Figure 1B, cells in G1, 53\% $3 \times A B F 2^{+}$compared 


\section{Author Summary}

How cells determine when to divide is critical for understanding biological processes where cell proliferation is manifest. Because cells need to accumulate precursors prior to duplication, cellular metabolism is expected to impact cell division. Mitochondrial processes are central to the control of overall cell metabolism. Yet, the mechanisms that link mitochondrial processes with nuclear DNA replication remain largely unknown. We found that budding yeast cells moderately over-expressing Abf2p, a mtDNA maintenance protein, accelerate nuclear DNA replication. These cells with more mitochondrial DNA proliferate and increase in size more rapidly than their wild type counterparts. The results suggest that cells overexpressing Abf2p have up-regulated metabolic functions, which in turn enable these cells to accelerate initiation of cell division. We also examined the role of Sir2p, an NAD ${ }^{+}$ dependent de-acetylase, which negatively controls DNA replication. We found that the level of Sir $2 p$ bound at origins of DNA replication is inversely related to the amount of mtDNA in the cell. In summary, our findings challenge the notion that metabolic processes are required for cell division by simply operating at constitutive background levels. Instead, our work suggests that mitochondrial transactions can actively promote DNA replication and cell division.

to $61 \% A B F 2^{+}$). In contrast, under $\mathrm{N}$-limitation, extra copies of $A B F 2$ did not affect the DNA content distribution in anabolicallyrestricted cells (Figure 1B, cells in G1, 52\% $3 \times A B F 2^{+}$compared to $\left.51 \% A B F 2^{+}\right)$. These data suggest a connection between mitochondrial function and cell cycle progression that is evident under glucose limitation in cells over-expressing $A B F 2$. Interestingly, $3 \times A B F 2^{+}$cells are the same size as wild type cells (Figure 2A), possibly explaining why $A B F 2$ mutations were not identified previously in size-based mutant screens for cell cycle regulators.

To determine whether the effects observed in cell cycle progression are due to $A B F 2$ and not due to an unknown secondary mutation, we repeated the experiment shown in Figure 1B using a different genetic background (BY4743). We used a low-copy centromeric plasmid carrying $A B F 2\left(C E N-A B F 2^{+}\right)$to achieve modest over-expression of Abf2p similar to $3 \times A B F 2^{+}$cells $[9,20]$. Under glucose limitation, a lower fraction of $C E N-A B F 2^{+}$transformants $(45 \pm 1 \%)$ was in the $\mathrm{Gl}$ phase of the cell cycle compared to the empty vector transformants $(52 \pm 2 \%$, Figure $2 \mathrm{~B})$. Thus, the cell cycle effects we observed in $3 \times A B F 2^{+}$cells are $A B F 2$-linked.

\section{Abf2p and the Retrograde Response}

If mitochondria do not function properly a retrograde (RTG) response leads to elevated ( $\sim 10$-fold) CIT2 levels [21,22]. As expected, in $\rho^{\circ}$ cells, which lack mtDNA, the CIT2 RNA level was increased $\sim 5$-fold over the level in $\rho^{+}$cells (Figure 3 ). In contrast, we found that CIT2 mRNA levels are not elevated in cells overexpressing ABF2. Instead, CIT2 levels are reduced by $\sim 2$-fold (Figure 3). Thus, the mitochondria of cells over-expressing ABF2 are not dysfunctional. Using a colony sectoring assay [23], we also found that the frequency of chromosome loss was $1.66 \%$ $(\mathrm{n}=3,004)$ for $C E N-A B F 2^{+}$transformants, compared to $1.73 \%$ $(n=2,657)$ for the empty vector transformants. Therefore, overexpression of $A B F 2$ does not cause gross genome instability.

\section{Abf2p and the Timing of START}

Because $3 x A B F 2^{+}$populations proliferate faster (Figure 1A) and have a reduced fraction of G1 cells (Figure 1B), we hypothesized
A

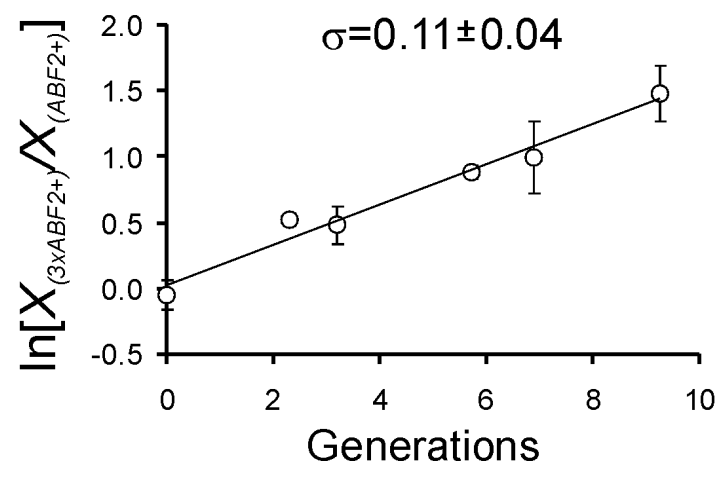

B

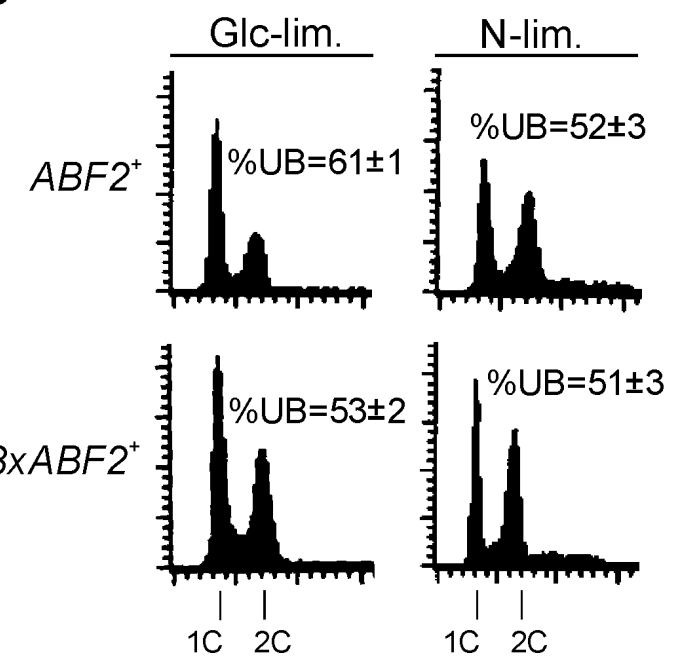

Figure 1. Cells moderately over-expressing $A B F 2$ proliferate faster $(A)$, and have altered cell cycle progression $(B)$ in chemostat cultures. In $A$, a chemostat competition experiment done during glucose limitation at dilution rate $D=0.1 \mathrm{~h}^{-1}$ is shown. The specific growth differential $(\sigma)$ between the two competing strains [41] is the average ( \pm s.d.) of three independent competition experiments. In each experiment, and for each sampling point, the average ( \pm s.d.) of at least three measurements was calculated. $\mathrm{X}_{3 \times A B F 2}{ }^{+}(\mathrm{t})$ and $\mathrm{X}_{A B F 2}{ }^{+} \mathrm{t}$ ) represent the relative proportion of the two strains at time $t$, in generations. In $B$, cell cycle progression of the indicated strains and nutrient limitations was evaluated by flow cytometry. Cell numbers are plotted on the $y$-axis and fluorescence on the $x$-axis. The DNA content of cells in $\mathrm{G} 1$ and $\mathrm{G} 2 / \mathrm{M}$ is indicated as $1 \mathrm{C}$ and $2 \mathrm{C}$, respectively. The percentage of unbudded cells (\%UB) correlates with $\mathrm{G} 1$ cells. The values shown are the average ( \pm s.d.) of at least three independent experiments.

doi:10.1371/journal.pgen.1000047.g001

that DNA replication may be accelerated in $3 \times A B F 2^{+}$cells. We examined cell cycle progression of $A B F 2^{+}$and $3 x A B F 2^{+}$cells from synchronous cultures obtained by centrifugal elutriation. We used standard [24] undefined medium (YPD) for these experiments, with lower glucose concentration $(0.5 \%)$. A higher fraction of $3 \times A B F 2^{+}$cells entered $\mathrm{S}$ phase sooner than $A B F 2^{+}$cells (Figure 4, compare the top two rows). For example, at $60 \mathrm{~min}$ postelutriation of $A B F 2^{+}$cells $10.7 \%$ were budded and $46.2 \%$ in $\mathrm{G} 1$, while of $3 \times A B F 2^{+}$cells $40.2 \%$ were budded and $32 \%$ were in $\mathrm{G} 1$. In addition, the $3 \times A B F 2^{+}$cells completed $\mathrm{S}$ phase sooner than $A B F 2^{+}$cells (see Figure 4 compare the top two rows): At $80 \mathrm{~min}$ post elutriation, note the size of the G2/M peak relative to the $\mathrm{G} 1$ 
A

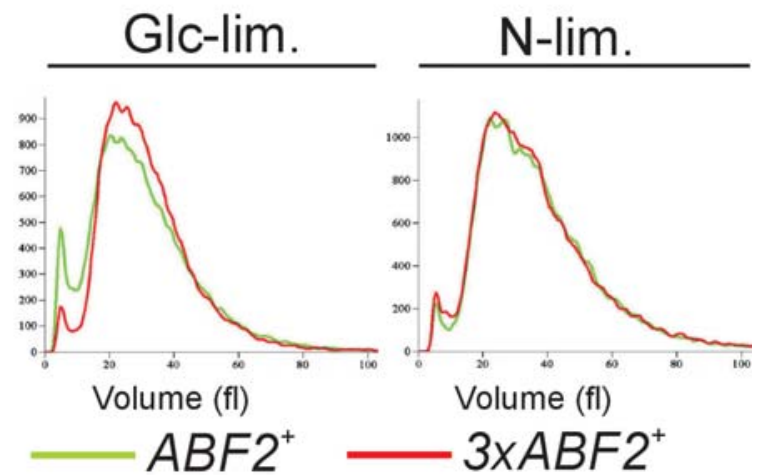

B

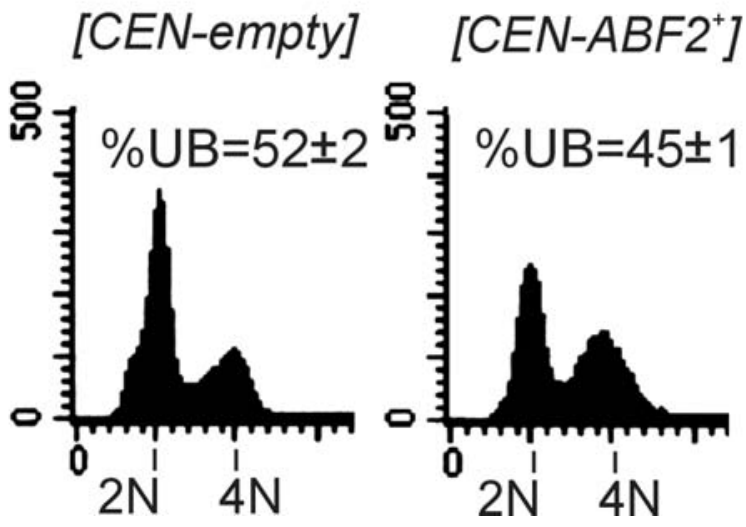

Figure 2. $3 x \mathrm{ABF}^{+}$cells do not have altered cell size in chemostat cultures. $A$, The cell size of the indicated cell populations was measured from the same chemostat experiments described in Figure $1 B$, using a channelyzer. Cell numbers are plotted on the $y$-axis and the $\mathrm{x}$-axis indicates size (in $\mathrm{fl}$ ). $B$, Moderate over-expression of $A B F 2$ from a low-copy CEN plasmid promotes cell cycle progression. The DNA content of the indicated strains is shown.

doi:10.1371/journal.pgen.1000047.g002

peak. More $3 x A B F 2^{+}$cells have completed DNA replication than $A B F 2^{+}$cells. Finally, although in asynchronous populations the overall cell size of $3 \times A B F 2^{+}$cells was not different from $A B F 2^{+}$cells (Figure 2A), the elutriated daughter $3 x A B F 2^{+} \mathrm{G} 1$ cells increased in size faster than their $A B F 2^{+}$counterparts (Figure 4, compare the top two rows): at $60 \mathrm{~min} 3 \times A B F 2^{+}$cells are $40.2 \mathrm{fl}$, while $A B F 2^{+}$ cells are $37.1 \mathrm{fl}$, consistent with a growth-promoting role of Abf2p.

To confirm these results, we repeated this analysis several times. In each case, $A B F 2^{+}$and $3 \times A B F 2^{+}$cells were examined under identical conditions, using media from the same batch. We used two variables to compare the two strains across different experiments: the critical size for budding (at which 50\% of the cells are budded); and the rate of cell size increase after elutriation. Interestingly, $3 \times A B F 2^{+}$cells bud at a slightly larger size $(41.2 \pm 1.1$ $\mathrm{fl}, \mathrm{n}=5, P=0.032$, based on a 2-tailed Student's $t$ test) than $A B F 2^{+}$ cells $(38.6 \pm 1.1 \mathrm{fl}, \mathrm{n}=6$ ) (Figure S1A and S1D). We then plotted in each case cell size as a function of time, to estimate the rate of cell size increase (fl/min) after elutriation (Figure S1B and S1C). While these values can vary from experiment to experiment, in every case $3 \times A B F 2^{+}$cells increased in size significantly faster $(0.14 \pm 0.02$ $\mathrm{fl} / \mathrm{min}, \mathrm{n}=5, P=0.013$, based on a 2-tailed Student's $t$ test) than $A B F 2^{+}$cells $(0.11 \pm 0.01 \mathrm{fl} / \mathrm{min}, \mathrm{n}=6)$ (Figure $\left.\mathrm{S} 1 \mathrm{E}\right)$. Thus, even though $3 \times A B F 2^{+}$cells have a slightly larger critical size for budding than $A B F 2^{+}$cells, they reach that size significantly earlier than

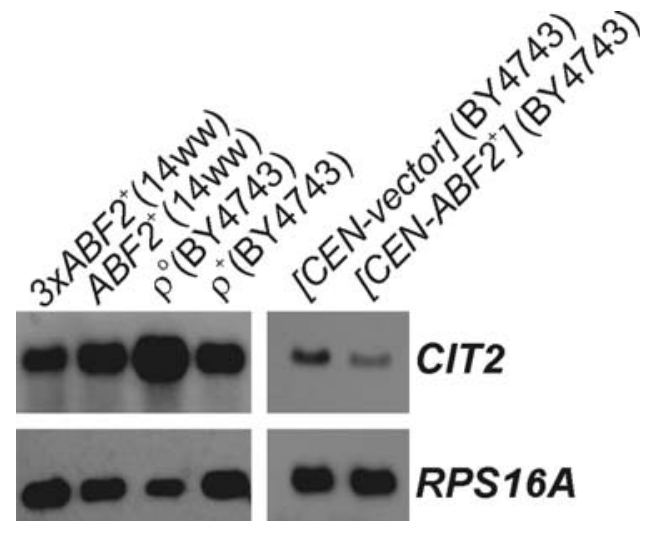

Figure 3. Moderate over-expression of $A B F 2$ does not trigger the RTG response. RNA blots of CIT2 steady-state levels, from cells grown under glucose limitation in chemostats. The relevant genotype and strain background are shown above each lane. RPS16A levels indicate loading.

doi:10.1371/journal.pgen.1000047.g003

their $A B F 2^{+}$counterparts because they increase in size $\sim 28 \%$ faster. For example, for $A B F 2^{+}$newborn daughter cells of $20 \mathrm{fl}$, it will take on average 169 min until they reach their critical size, but it will take $150 \mathrm{~min}$ for $3 \times A B F 2^{+}$daughters. Together with our chemostat experiments (Figures 1 and 2), our findings from these synchronous cultures (Figure 4 and Figure S1) with standard YPD media strongly support the notion that Abf2p plays an active growth-promoting role and accelerates initiation of DNA replication.

We also examined the levels of the cyclin-dependent kinase (Cdk) inhibitor Siclp in cells over-expressing Abf2p. In late G1 rising levels of Cdk activity trigger the degradation of Siclp and initiation of DNA replication [25]. In cells over-expressing Abf2p degradation of Siclp was initiated sooner than in the control cells (Figure 5), consistent with a shortened G1 phase, but once triggered the rate of Siclp degradation was unaffected. We obtained identical results in separate repeats of this experiment (Figure S2).

We next generated the corresponding $\rho^{-}$strains to test whether over-expression of $\mathrm{ABF}^{+}$requires mtDNA to promote DNA replication. These strains are respiratory incompetent (Figure S4A). DNA replication was not accelerated in $3 x A B F 2^{+}\left(\rho^{-}\right)$cells (Figure 6A). Overall, in contrast to $\rho^{+}$cells (see Figure 4 and Figure $\mathrm{S} 1$ ) the critical budding size (Figure S4B), and the rate of cell size increase after elutriation (Figure $\mathrm{S} 4 \mathrm{C}$ ), were not significantly different between $A B F 2^{+}\left(\rho^{-}\right)$and $3 \times A B F 2^{+}\left(\rho^{-}\right)$cells: $P=0.43$, and $P=0.54$, respectively (based on 2-tailed Student's $t$ tests). In conclusion, our findings suggest that altered mitochondrial functions in $3 \times A B F 2^{+}$cells impact on some factor(s) that affect DNA replication.

\section{Functional Interactions between Abf $2 p$ and Sir $2 p$}

A protein linked to both metabolism and DNA replication is the Sir2p sirtuin [11], which negatively impacts DNA replication [1618]. Consequently, we evaluated cell cycle progression of cells lacking Sir2p alone, or in combination with Abf2p over-expression (Figure 4). Comparison of $A B F 2^{+}, \operatorname{SIR}^{+}$(Figure 4, top row) to $A B 2^{+}, \operatorname{sir} 2 \Delta$ (Figure 4, third row) cells at 60 min shows that cells lacking SIR2 initiated and completed S phase significantly sooner than wild type cells. Initiation of DNA replication was further accelerated in $3 x A B F 2^{+}$, sir $2 \Delta$ cells (Figure 4 , bottom row). We repeated this analysis several times, as we described earlier (Figure 


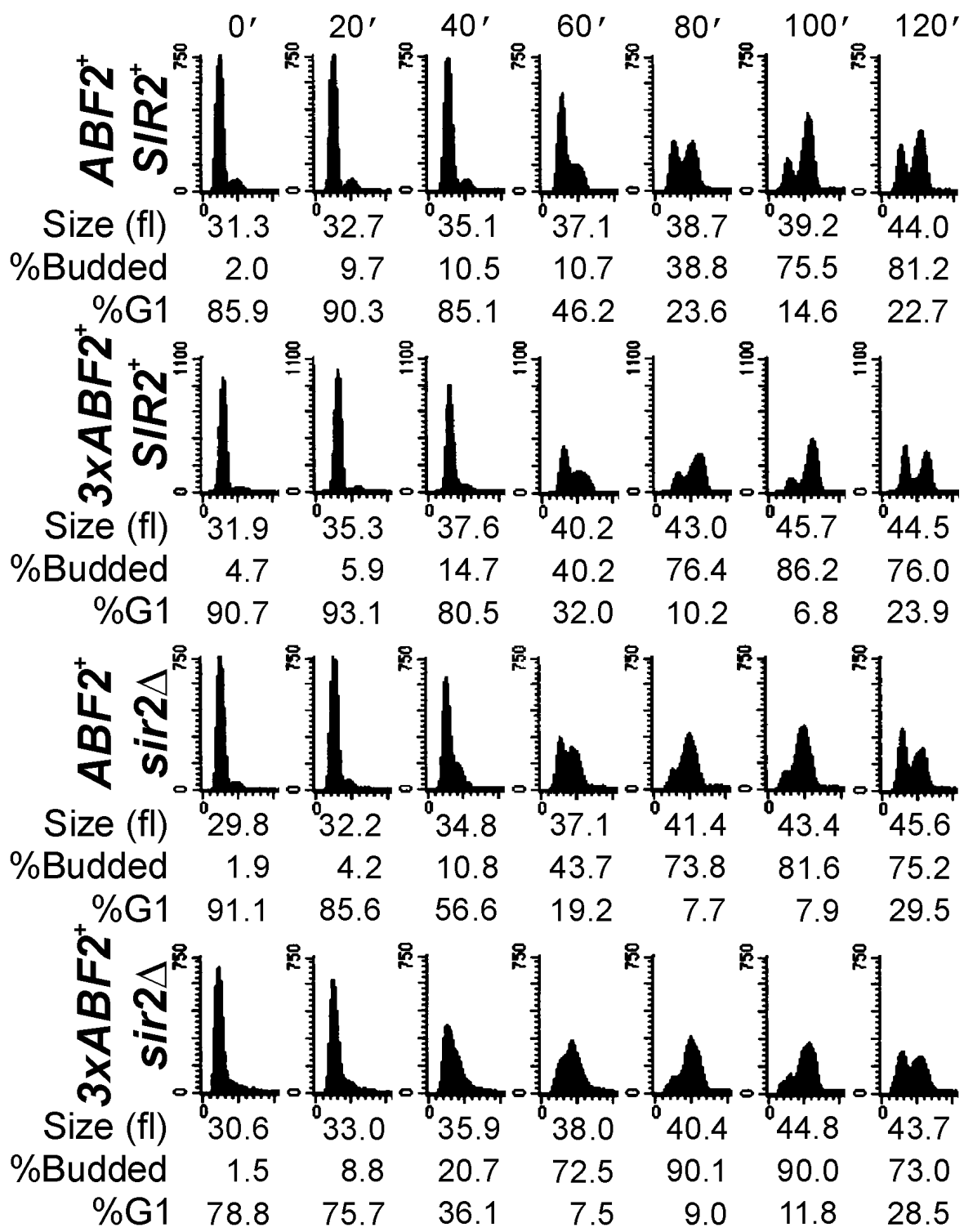

Figure 4. Over-expression of $A B F 2$, or loss of $S I R 2$, accelerates DNA replication. Synchronous cultures of the indicated strains were obtained by centrifugal elutriation and the starting populations of each strain were of the same size. At the indicated times the DNA content was evaluated by flow cytometry.

doi:10.1371/journal.pgen.1000047.g004

S1). Interestingly, $3 x A B F 2^{+}$, sir $2 \Delta$ cells bud at a smaller size (36.1 $\pm 0.5 \mathrm{fl}, \mathrm{n}=5, P=0.0009$, based on a 2-tailed Student's $t$ test) than $A B F 2^{+}, S I R 2^{+}$cells $(38.6 \pm 1.1 \mathrm{fl}, \mathrm{n}=6$ ) (Figure S1D). This explains the apparent additive acceleration of START we observed in $3 \times A B F 2^{+}$, sir $2 \Delta$ cells (Figure 4 , compare at $40 \mathrm{~min}$ the $3 \times A B F 2^{+}, \operatorname{sir} 2 \Delta$ strain to other strains, and see also Figure 7 , below). Taking into account the critical budding size and the rate of cell size increase for each strain, for $3 \times A B F 2^{+}$, sir $2 \triangle$ newborn daughter cells of $20 \mathrm{fl}$, it will take on average $128 \mathrm{~min}$ to start budding, compared to $169 \mathrm{~min}$ for $A B F 2^{+}, S I R 2^{+}$daughters. Finally, loss of Sir2p does not increase the amount of mtDNA in the cell (Figure S3).

We next generated the corresponding $\left(\rho^{-}\right)$strains lacking Sir2p (Figure S4A). These strains were examined after elutriation (Figure S4B and S4C), as we described above. Strains lacking Sir2p and mtDNA did not have a significant different rate of cell size increase after elutriation compared to the other $\left(\rho^{-}\right)$strains (Figure S4C). Finally, to ensure that the effects of Sir $2 p$ on cell cycle progression were not strain-specific, we also examined cell cycle progression of sir $2 \Delta$ cells in a different strain background (an S288C derivative) [14]. S phase entry was greatly accelerated in $\operatorname{sir} 2 \Delta$ cells in that background, and cells spent very little time in G1 (Figure 6B). For example, $S I R 2^{+}$cells initiate DNA replication at 80-100 min after elutriation, while $\operatorname{sir} 2 \Delta$ cells do so at $\sim 40 \mathrm{~min}$.

We next tested if the acceleration of DNA replication in $3 x A B F 2^{+}$or sir $2 \Delta$ cells depends on NADH. Yeast cells can display robust $\mathrm{NAD}(\mathrm{P}) \mathrm{H}$ oscillations, which are thought to gate metabolism with DNA replication, since DNA synthesis takes place when $\mathrm{NAD}(\mathrm{P}) \mathrm{H}$ levels are high [26-28]. To deplete cellular NADH we added $10 \mathrm{mM}$ acetaldehyde to the elutriated early-G1 cells [29]. The G1 phase was greatly expanded (Figure 7), compared to the untreated cells shown in Figure 4, consistent with a requirement for $\mathrm{NADH}$ for initiation of DNA replication. G1 phase expansion was also evident in $3 x A B F 2^{+}, S_{R} 2^{+}$or $A B F 2^{+}$, sir2 $\triangle$ cells, indicating that these cells still require $\mathrm{NADH}$ to progress through G1 into $\mathrm{S}$ phase. Nonetheless, $3 \times A B F 2^{+}, S I R 2^{+}$or $A B F 2^{+}$, sir $2 \triangle$ 

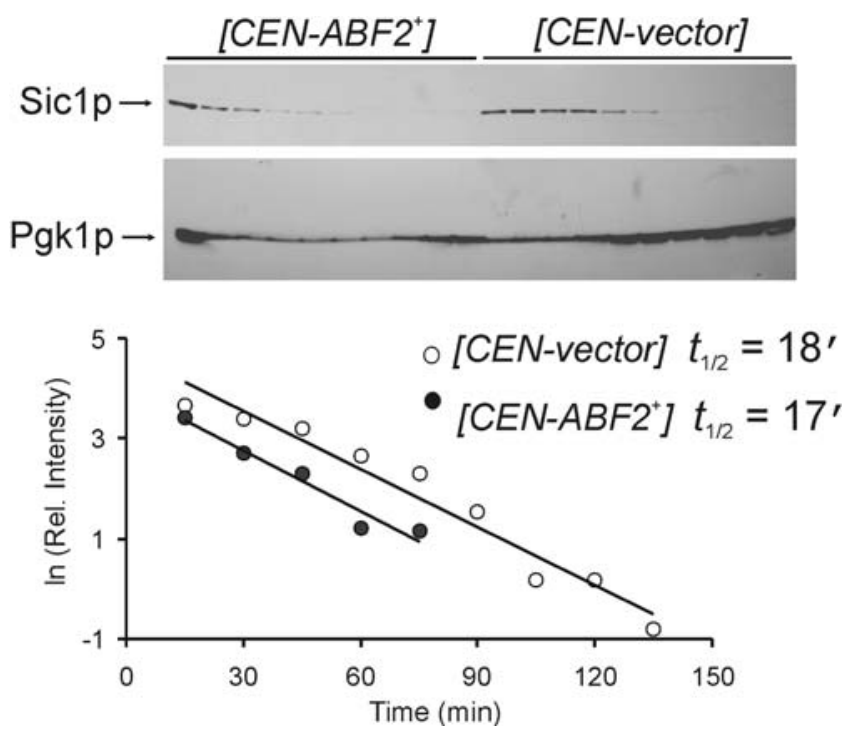

Figure 5. Sic1p stability and Abf2p. The levels of galactose-induced Sic1p-TAG were monitored by immunoblotting from cells carrying the indicated plasmids after SIC1-TAG expression was turned off. The signal from Pgk1p was used to estimate loading.

doi:10.1371/journal.pgen.1000047.g005

cells entered $\mathrm{S}$ phase sooner $(\sim 20 \mathrm{~min})$ than wild type $A B F 2^{+}$, $S I R 2^{+}$cells (Figure 7, compare the top three rows at $140 \mathrm{~min}$ postelutriation), consistent with our previous results shown in Figure 4. Remarkably, $3 \mathrm{x} A B F 2^{+}$, sir $2 \Delta$ cells entered and completed $\mathrm{S}$ phase with highly accelerated kinetics: they finished DNA replication before wild type cells even started (Figure 7, compare the bottom row with the top row). These results are consistent with strong additive functional interactions between Abf2p and Sir2p, with Sir2p acting antagonistically to Abf2p's effects on DNA replication. How Abf2p over-expression impacts the metabolic status of the cell is unclear, but it may involve NAD/NADH metabolism because the functional interactions between Abf2p and Sir2p are quite prominent in the presence of acetaldehyde.

We next asked if Sir2p negatively affects cellular metabolism to delay DNA replication. We found that sir2 $\Delta$ cells did not proliferate faster than $S I R 2^{+}$cells under glucose limitation in chemostats (Figure 8). Thus, loss of Sir2p does not up-regulate metabolic functions necessary to achieve the proliferation advantage evident in $3 \times A B F 2^{+}$cells under the same conditions (Figure 1A).

\section{Abf2p and Physical Changes at Origins of DNA Replication}

The overall levels of Sir2p are not altered in $3 \times A B F 2^{+}$cells (Figure 9A). In addition to its roles in silencing, Sir2p negatively affects the activity of origins of DNA replication throughout the genome $[16,17]$. Consequently, we next tested if the level of Sir2p at origins of DNA replication is altered in $3 \times A B F 2^{+}$cells.

We examined the ARS elements in the rDNA tandem repeats, because the association of Sir2p with the rDNA [30] and the negative role of Sir2p in regulating these origins $[16,17]$ are wellcharacterized. Chromatin immunoprecipitation (ChIP) was performed with $A B F 2^{+}$or $3 \times A B F 2^{+}$cells using antisera against Sir2p. $A B F 2^{+}$, sir2 $\triangle$ cells and $3 x A B F 2^{+}, \operatorname{sir} 2 \Delta$ cells were examined to provide a measurement of background. Immunoprecipitated DNA was analyzed by real-time PCR using primers that span the ARS elements in the rDNA. We found that the level of Sir2p at the rDNA ARS elements was reduced about two-fold in $3 \times A B F 2^{+}$cells, compared to the level in $A B F 2^{+}$cells (Figure 9B). The level of Sir2p at RPS16A, a locus that does not contain an ARS element, was not altered by over-expression of Abf2p. Consistent with the reduced level of Sir2p at the rDNA ARS elements, we also found that the level of K9, K14-acetylated histone $\mathrm{H} 3$ at the rDNA ARS elements was increased in cells over-expressing Abf2p (Figure 9G). In yeast and animals such chromatin modifications activate DNA replication [18,19,31]. In addition to the rDNA ARS we also examined ARS315, which is a very active origin and fires in $90 \%$ of the cell cycles [32]. Consistent with the high activity of ARS315, the level of K9, K14-acetylated histone H3 was also very high at that origin (data not shown). Since loss of Sir2p suppresses replication defects of ARS315 in $c d c 6-4$ cells [17], we then examined if Sir2p is present at ARS315. We did not detect Sir2p at ARS315 in $A_{B F 2}{ }^{+}$or $3 \times A B F 2^{+}$cells (Figure S5), perhaps consistent with the already high activity of this origin. Thus, the previously observed [17] effects of Sir2p on MCM proteins binding at ARS315 maybe indirect.

To answer if binding of Sir2p at the rDNA origins depends on the presence of mtDNA, we then examined the corresponding $\rho^{-}$ strains (Figure S6). Notably, in both $A B F 2^{+}$or $3 x A B F 2^{+} \rho^{-}$cells Sir2p levels are increased at the rDNA ARS by $\sim 2$-fold (Figure S6). Thus, the level of Sir2p bound at the rDNA ARS elements is inversely related to the amount of mtDNA in the cell. It appears that some mitochondrial function that depends on mtDNA limits the association of Sir $2 p$ with origins of replication. Together, these results identify physical changes associated with active origins of DNA replication in the nucleus, resulting from an increase in the amount of the mitochondrial genome (Figure 10).

In conclusion, the control of DNA replication by an increase in mtDNA we describe here suggests that the mitochondrion does not simply provide the energy at the service of its larger cellular host, but it may actively dictate when cells initiate their division. Furthermore, metabolic control of chromatin modifications may provide critical links between metabolism and cell division.

\section{Materials and Methods}

\section{Strains and Plasmids}

The haploid $3 x A B F 2^{+}$strain and its wild-type counterpart (14ww) have been described elsewhere [9]. Replacement of SIR2 with a sir2 $\Delta::$ KanMX6 cassette was done by standard methodology [33]. BY4743 is a standard diploid strain [34]. The strains used in Figure 3 were made $\rho^{\circ}$ after three passages of single colonies on plates containing $25 \mu \mathrm{g} / \mathrm{ml}$ ethidium bromide. A single colony from the final ethidium bromide passage was then plated on glucose and glycerol-containing plates to ensure the isolated colony was respiratory deficient and no growth occurred on the glycerol plates. Further PCR analysis failed to detect the presence of the COX2 gene, which is mtDNA-encoded, in the $\rho^{\circ}$ strains, but it detected the CIT2 gene, which is nDNA-encoded. We similarly generated the $\rho^{-}$strains used in Figure 6A, Figure S4, and Figure S6.

For the Siclp stability experiment described in Figure 5 and Figure S2, the strains used were diploids, obtained from a cross of YSC3869-9515050 ([P ${ }_{G A L}-S I C 1-T A G:: U R A 3$ [35]], BY4741 [34] otherwise; purchased from Open Biosystems), with strain BY4742 [34] carrying $\left(C E N-A B F 2^{+}\right)[9,20]$, or the empty vector $(C E N-$ vector).

\section{Yeast Cultivation}

For batch cultures we followed established yeast protocols [24]. Conditions for chemostat cultures have been described previously $[36,37]$. 
A

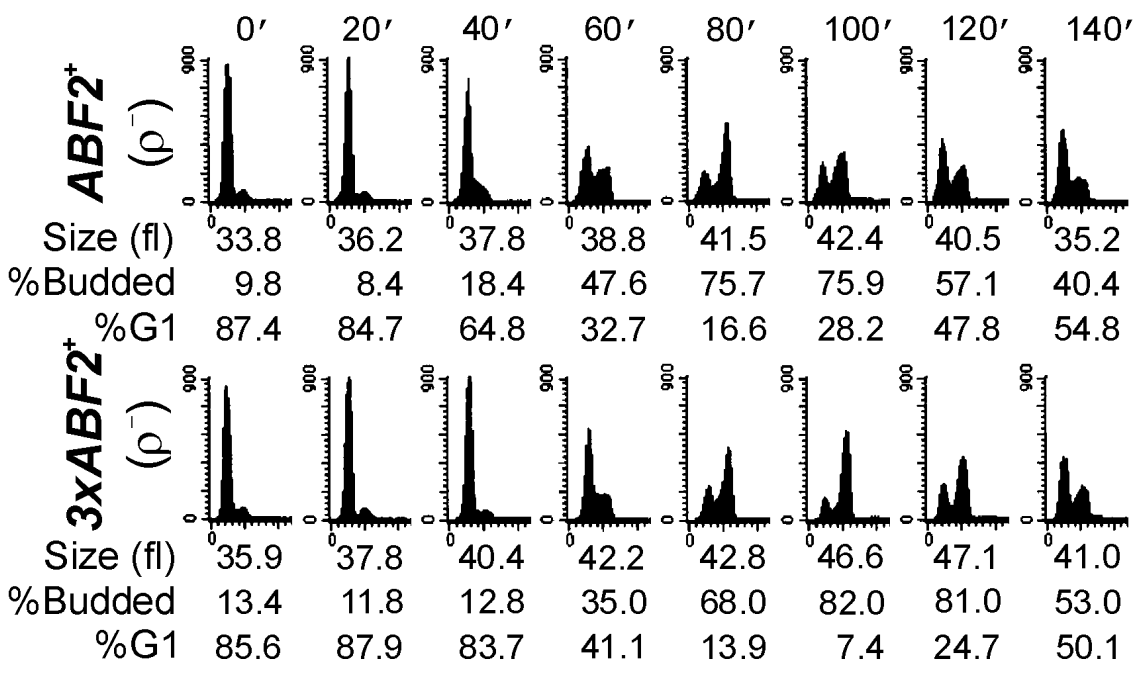

B

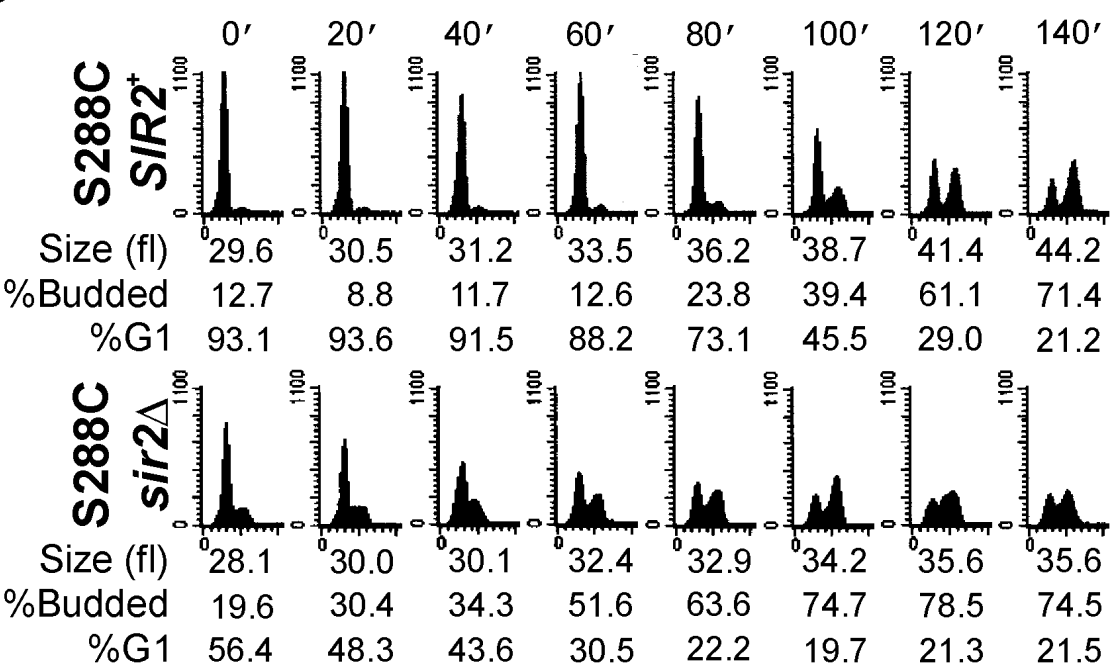

Figure 6. mtDNA is required for the accelerated DNA replication in $3 \times A B F 2^{+}$cells. $A$, Cell cycle progression of elutriated cells was analyzed exactly as in Figure 4, except that the strains used were made $\rho^{-}$as described in the Methods. B, Loss of SIR2 accelerates DNA replication in the S288C strain background. The elutriation experiment was done as in Figure 4, except that the strains used were in a different strain background, described previously [14].

doi:10.1371/journal.pgen.1000047.g006

\section{Elutriation}

We followed previously described protocols to obtain cell cycle parameters [38], except that the cells were cultured and collected in YPD $(0.5 \%$ glucose $)$ medium. The percentage of cells in G1 from the flow cytometry panels was calculated from the DNA histograms using the ModFit ${ }^{\circledR}$ software (Verity Software House, $\mathrm{ME}$ ). Cell cycle progression was also monitored microscopically, by the percentage of budded cells. Cell size (fl) was measured using a channelyzer.

From each elutriation experiment, we plotted the percent of budded cells as a function of cell size. To estimate the critical budding size, when $\sim 50 \%$ cells are budded, we used data points from the linear portion of each graph, which were fit to a straight line using the regression function in Microsoft Excel. From the resulting equation $[(\%$ budded $)=a($ Cell size $)-b$; where $a$ is the slope and $\mathrm{b}$ the $\mathrm{y}$-intercept of the line] we calculated the critical budding size for each experiment. The average of all experiments for each strain was then calculated, along with the associated standard deviation.

From the same elutriations, to calculate the rate of cell size increase, we plotted the cell size as a function of time. The data were also fit to a straight line using the regression function in Microsoft Excel. From the slope of the line we obtained the rate of cell size increase. The average of all experiments for each strain was then calculated, along with the associated standard deviation.

\section{RNA Methods}

Standard protocols [24] were used for RNA extraction and electrophoresis. The CIT2 and RPS16A probes (see Figure 3) were generated by PCR, and labeled using non-radioactive reagents 


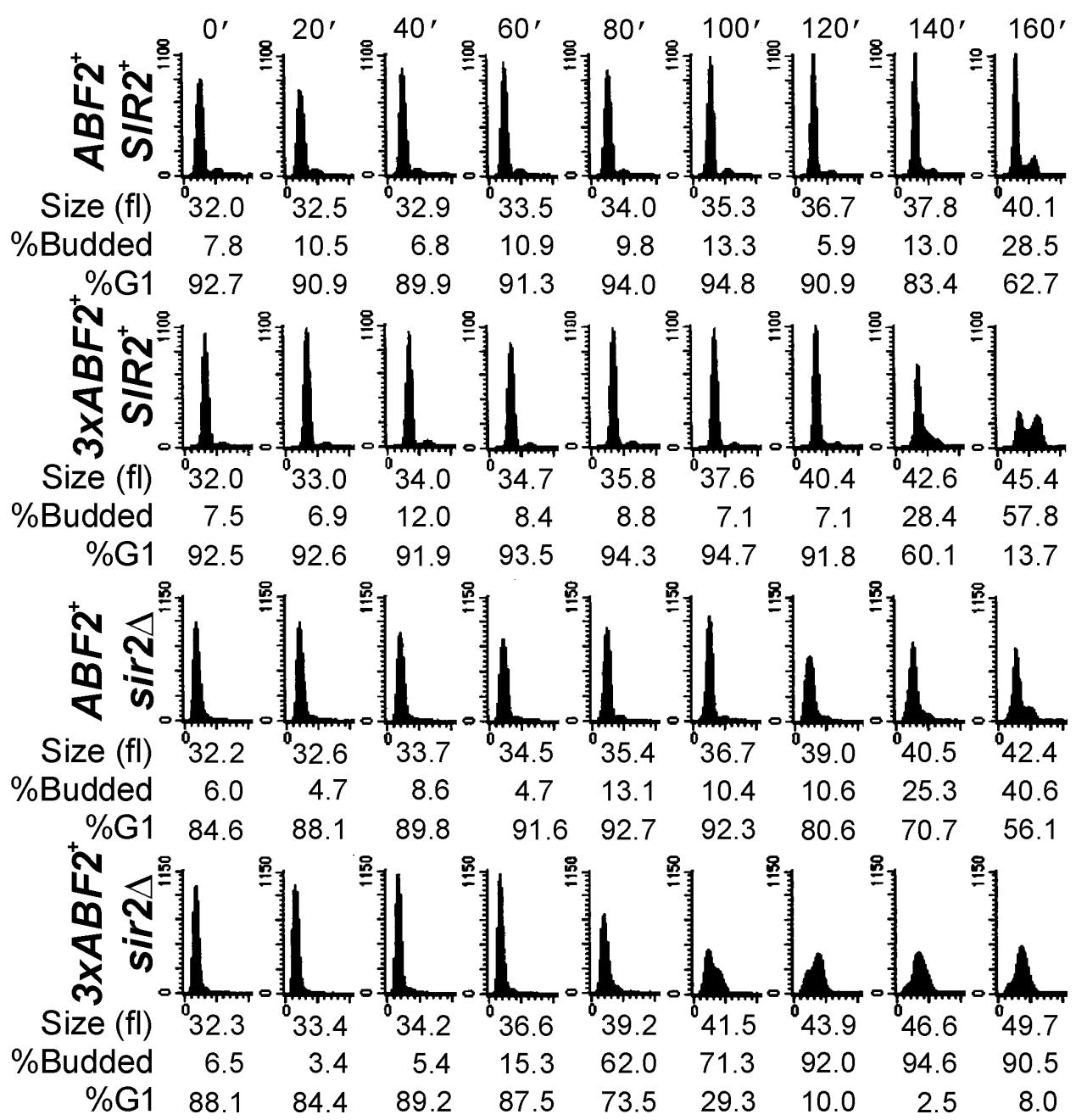

Figure 7. Loss of SIR2 in cells over-expressing ABF2 dramatically accelerates DNA replication when NADH is depleted. Cell cycle progression was monitored after elutriation as in Figure 4, except that $10 \mathrm{mM}$ acetaldehyde was added to the starting samples. doi:10.1371/journal.pgen.1000047.g007

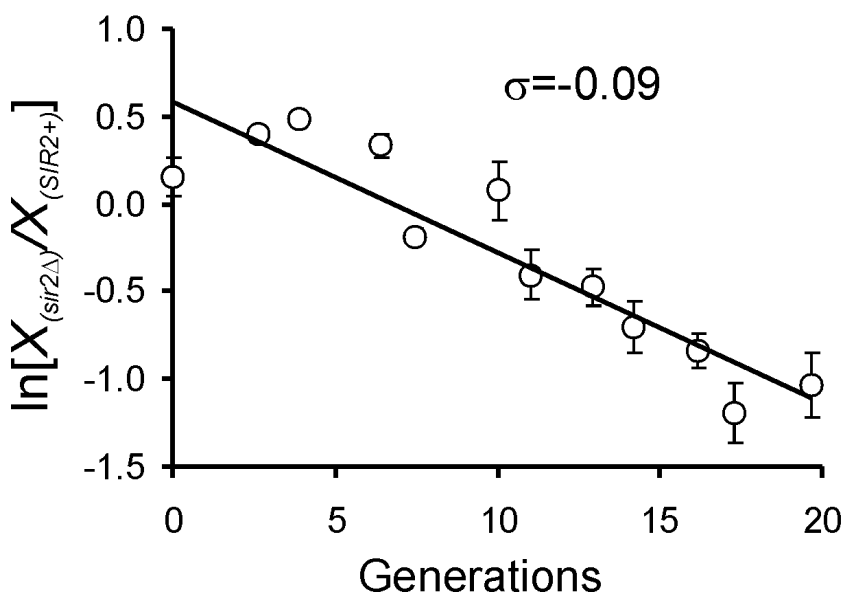

Figure 8. Loss of Sir2p does not accelerate overall cell proliferation. Chemostat competition experiments between $S I R 2^{+}$ and sir2 $\Delta$ cells (in the $14 \mathrm{ww}$ strain background) were done during glucose limitation at dilution rate $D=0.1 \mathrm{~h}^{-1}$, as described in Figure 1 . doi:10.1371/journal.pgen.1000047.g008 from the North2South ${ }^{\circledR}$ Biotin Random Prime Labeling Kit (Pierce), according to their instructions. Probe hybridization and detection were performed according to the North2South ${ }^{\circledR}$ Chemiluminescent Hybridization and Detection Kit (Pierce).

mtDNA Abundance

We used PCR to estimate the relative amount of mtDNA (see Figure S3). The exponential range of amplification for the COX2 (mtDNA-encoded) and GID 8 (nDNA-encoded) PCR products were determined by performing separate reactions for each of the two amplified products and removing them after 25, 30, or 35 cycles. The PCR products were run on a $2 \%$ agarose gel and the ethidium-stained signal intensities for the 30 -cycle products (which were in the linear range of amplification) were quantified with Adobe Photoshop ${ }^{\circledR}$. The ratio between the COX2 and the GID 8 product was determined to normalize for differences in initial DNA concentration and reaction efficiencies. The ratio for each strain was then compared to $A B F 2^{+}$cells to determine the relative amounts of mtDNA.

\section{Sic1p Stability Assays}

Cultures were diluted 10-fold from an overnight culture in selective liquid synthetic complete medium containing raffinose as 

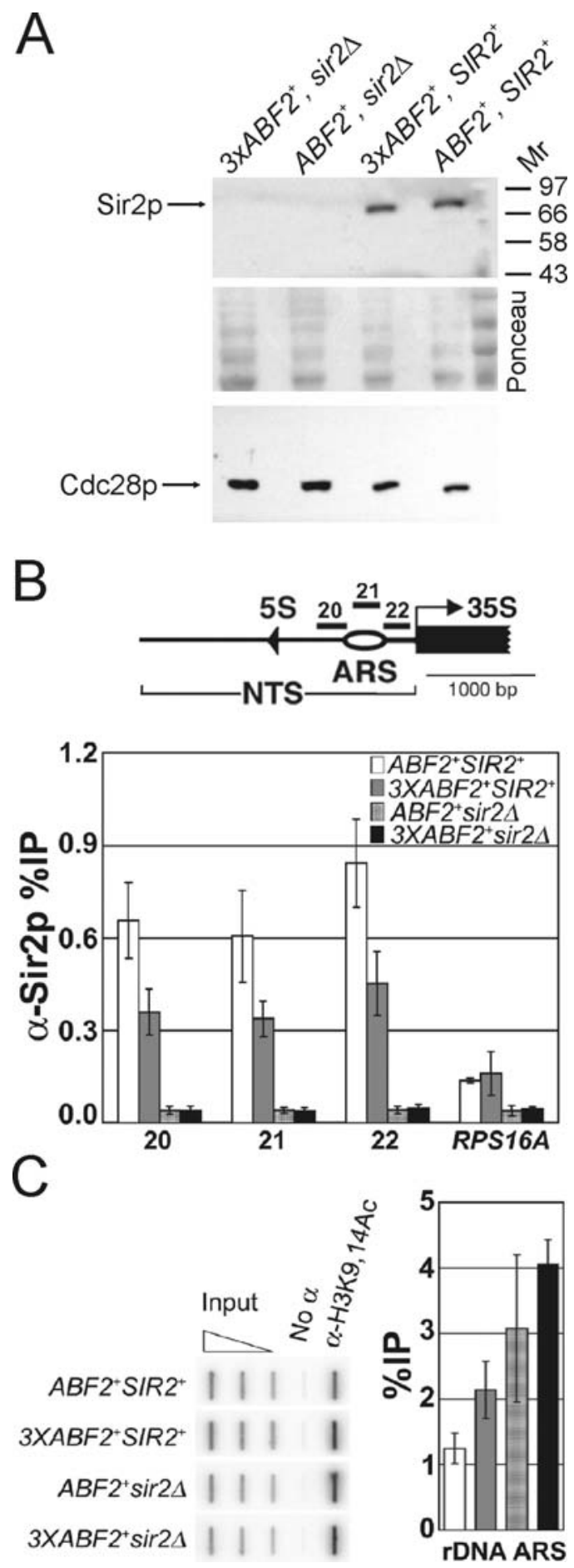

Figure 9. Cells over-expressing $A B F 2$ have less Sir2p and higher levels of K9, K14 acetylated histone $\mathrm{H3}$ at the rDNA ARS elements. $A$, Immunoblot showing that the level of Sir $2 p$ is not altered in whole cell extracts from cells over-expressing ABF2. The same blot was stained with Ponceau, to indicate loading. Loading was also evaluated from the same samples, with an anti-Cdc28p antibody. $B$, ChIP experiments analyzed by real-time PCR show that the level of Sir $2 p$ (\%IP) at the rDNA ARS elements is reduced in $3 X A B F 2^{+}$cells. Part of one rDNA repeat is shown above indicating the location of the rDNA ARS elements, the primers used (primer pairs 20,21,22); the nontranscribed spacer (NTS); and the 35S and 5S rRNA genes. The values shown in the bar graph are the average \%IPs ( \pm s.d.) of three independent experiments. $C$, ChIP experiments analyzed by slot blot show that the level of $\mathrm{K} 9, \mathrm{~K} 14$ acetylated histone $\mathrm{H} 3$ is increased at the rDNA ARS elements in $3 X A B F 2^{+}$cells. In the graph, the average \%IPs (+/- range) are shown for two independent experiments (Pearson coefficient $=0.95$ ). The open triangle above the slot blot represents serial dilutions of input DNA to ensure linearity with respect to hybridization of the probe to the amount of DNA applied to the membrane. Other labels as in Figure 9B.

doi:10.1371/journal.pgen.1000047.g009

carbon source, to a total volume of $10 \mathrm{ml}$. Cells were grown at $30^{\circ} \mathrm{C}$, with shaking for 30-48 h to synchronize the cells in G1 by starvation. Galactose was then added to $2 \%(\mathrm{w} / \mathrm{v})$ final concentration for 2 to 3 hours to induce expression of $\mathrm{P}_{G A L^{-}}$ SIC1-TAG. After induction, the cells were pelleted, washed in water, and re-suspended in $20 \mathrm{ml}$ fresh medium containing $2 \%$ $(\mathrm{w} / \mathrm{v})$ glucose, to stop expression of $\mathrm{P}_{G A L}-S I C 1-T A G$. The cells were cultured at $30^{\circ} \mathrm{C}$ and $1 \mathrm{ml}$ was taken every $15 \mathrm{~min}$, to monitor Siclp-TAG levels. Proteins were extracted using TCA precipitation and resolved by SDS-PAGE. For immunoblotting, we used the PAP reagent (Sigma, used according to their instructions) to detect the Protein A epitope present in Siclp-TAG. The same blot was also probed with an anti-Pgklp antibody (from Molecular Probes, and used according to their instructions). Pgklp is not cell cycle regulated, and it is widely used as a loading control in cell cycle experiments, including landmark studies that accurately quantified the levels of cell cycle proteins [39]. The intensity of the bands was quantified using Adobe Photoshop, normalizing the levels of Siclp-TAG to the loading control. Using Microsoft Excel, values were fit to a linear transformation of the exponential decay equation $\left(\ln \mathrm{X}_{t}=-k t+\ln \mathrm{X}_{t}\right.$, where $\mathrm{X}$ denotes the amount of Siclp-TAG, $k$ the degradation rate constant, and $t$ time) to obtain $k$ from the slope of the line. The half-life of Siclp-TAG was then determined from $t_{1 / 2}=\ln 2 / k$.

\section{MITOCHONDRIA}

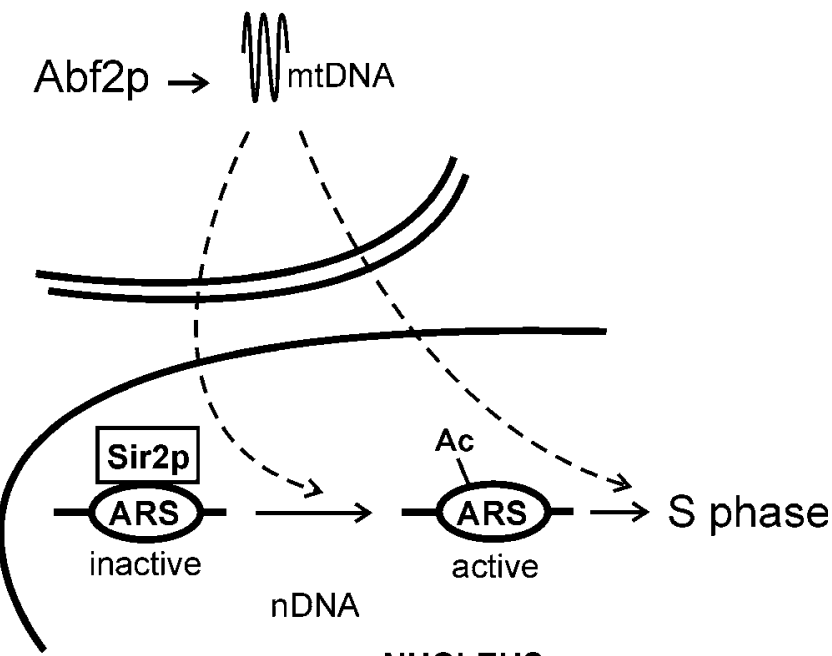

\section{NUCLEUS}

Figure 10. A schematic of the model suggested by our data. An increase of mtDNA by moderate over-expression of Abf $2 p$ promotes mitochondrial functions, which in turn accelerate cell proliferation and DNA replication. The $\mathrm{NAD}^{+}$-dependent de-acetylase Sir2p antagonizes initiation of DNA replication. Cells over-expressing Abf2 $p$ have decreased levels of Sir2p at origins of DNA replication (ARS), and higher levels of acetylated K9, K14 histone $\mathrm{H} 3$ residues (Ac). Additional mechanisms likely contribute to the overall positive effects on cell proliferation and DNA replication in cells with higher mtDNA levels. doi:10.1371/journal.pgen.1000047.g010 


\section{Chromatin Immunoprecipitation}

For these analyses, cells were cultured in YPD containing 0.5\% glucose. The primers and protocols for ChIP experiments and analysis have been described previously $[13,40]$. ChIPs were performed with anti-Sir2p antisera (Santa Cruz Biotechnology, Cat\#: sc-6666) or anti H3K9, K14Ac (Millipore, Cat\#: 06-599). Percent immunoprecipitations (\%IP) were determined by dividing IP signal by input signal. ChIP experiments were analyzed either by quantitative PGR (Figure 9B and Figure S5), or by slot blot (Figure 9C and Figure S6). For anti-Sir2p ChIPs, chromatin from sir $2 \Delta$ cells was analyzed to assess background signal. For anti H3K9, K14Ac ChIPs, "no antibody" controls were included to assess background signal. For slot blot analysis, samples were blotted to a membrane that was hybridized to a ${ }^{32} \mathrm{P}$-labeled probe spanning the rDNA ARS region. Signals were quantified on a Storm 860 phosphorimager (Molecular Dynamics) using Imagequant software. Ethidium-stained gels were quantified using Quantity One software (BioRad).

\section{Other}

For Sir2p immunoblotting (Figure 9A) we used anti-Sir2p antisera (Cat\#: sc-6666) from Santa Cruz Biotechnology, and a secondary antibody from Pierce, at the recommended dilutions. The anti-Gdc28p antibody used to estimate loading was also from Santa Cruz Biotechnology (Cat\#: sc-6708), and used according to their instructions. The immunoblots were processed with reagents from Pierce.

\section{Supporting Information}

Figure S1 Summary of data from elutriation experiments with rho $^{+}$strains. A, The raw data points showing the percent of budded cells as a function of cell size, from separate independent elutriation experiments with $\mathrm{ABF}^{+}$and $3 \mathrm{XABF} 2^{+}$strains. The data points shown were from the linear portion of each experiment, when the percentage of budded cells began to increase, and they were used to determine the critical budding size as described in Materials and Methods. B,C, The rate of cell size increase for each elutriation experiment of the indicated strains $\left(\mathrm{ABF}^{+}\right.$and $\left.3 \mathrm{XABF}^{+}\right)$is shown. From these graphs we calculated the rate of size increase as described in Materials and Methods. D, The critical size for budding of the indicated strains is shown. Where marked with an asterisk $\left(^{*}\right)$, the difference is statistically significant, based on 2-tailed Student's t tests. E, The rate of cell size increase of the indicated strains is shown. Where marked with an asterisk (*), the difference is statistically significant, based on 2-tailed Student's t tests.

Found at: doi:10.1371/journal.pgen.1000047.s001 (0.06 MB TIF)

Figure S2 Siclp stability and Abf2p. A separate experiment, similar to the one described in Fig. 5, is shown, except that loading was estimated from the Ponceau-stained blot.

\section{References}

1. Pringle JR, Hartwell LH (1981) The Saccharomyces cerevisiae cell cycle. In: Strathern JD, Jones EW, Broach JR, eds. The molecular biology of the yeast Saccharomyces. Cold Spring Harbor, NY: Cold Spring Harbor Laboratory Press. pp 97-142.

2. Ferea TL, Botstein D, Brown PO, Rosenzweig RF (1999) Systematic changes in gene expression patterns following adaptive evolution in yeast. Proc Natl Acad Sci U S A 96: 9721-9726.

3. Okamoto K, Shaw JM (2005) Mitochondrial morphology and dynamics in yeast and multicellular eukaryotes. Annu Rev Genet 39: 503-536.

4. MacAlpine DM, Perlman PS, Butow RA (1998) The high mobility group protein Abf2p influences the level of yeast mitochondrial DNA recombination intermediates in vivo. Proc Natl Acad Sci U S A 95: 6739-6743.
Found at: doi:10.1371/journal.pgen.1000047.s002 (0.23 MB TIF)

Figure S3 mtDNA abundance is not increased in cells lacking Sir2p. To estimate the mtDNA abundance of the indicated strains we used PCR (Top), as described in Materials and Methods. The ratio between the COX2 and the GID8 product was determined to normalize for differences in initial DNA concentration and reaction efficiencies. The ratio for each strain relative to $\mathrm{ABF}^{+}$ cells is shown (Bottom). Graph represents average data from two independent experiments ( $/-$ range). As a control, we also performed this analysis on $3 \mathrm{XABF}^{+}$cells, which are known to have higher mtDNA levels.

Found at: doi:10.1371/journal.pgen.1000047.s003 (0.11 MB TIF)

Figure S4 Cell cycle progression of rho $^{-}$strains. A, The strains used were respiratory-incompetent and they could not proliferate on plates with glycerol as a carbon source. The critical size for budding $(\mathrm{B})$, and the rate of cell size increase $(\mathrm{C})$, of the indicated strains was determined as in Figure S1.

Found at: doi:10.1371/journal.pgen.1000047.s004 (0.40 MB TIF)

Figure S5 ChIP experiments from the indicated strains analyzed by PGR do not detect Sir2p bound to ARS315 (left panel). As a control, we also performed this analysis on rDNA ARS using primer pair 21 and detected Sir2p association with the rDNA ARS (right panel). PCR products from input and IP samples were subjected to agarose gel electrophoresis and analyzed by ethidium bromide staining. The open triangles represent serial dilution of template DNA in the PGR reaction. Other labels as in Fig. 9B. Found at: doi:10.1371/journal.pgen.1000047.s005 (0.23 MB TIF)

Figure S6 Sir2p ChIP to rDNA ARS in $\mathrm{rho}^{+}$and rho ${ }^{-}$cells. ChIP experiments from the indicated strains analyzed by slot blot to detect Sir $2 p$ bound to rDNA ARS. Note that applying slot blot methodology to the rDNA ARS reproduced the reduced Sir2p levels bound to the rDNA ARS in $3 \mathrm{XABF}^{+}$cells that we observed with the real-time PGR analysis shown in Fig. 9B. Graph represents average data from two independent experiments $(+/$ - range). Other labels as in Fig. 9.

Found at: doi:10.1371/journal.pgen.1000047.s006 (0.26 MB TIF)

\section{Acknowledgments}

This paper is dedicated to the memory of Ron Butow. We thank R. Butow and G. Shadel for strains and plasmids, R. Butow and B. Kennedy for discussions, D. Pettigrew for advice and encouragement, and J. Kapler and D. Pettigrew for comments on the manuscript.

\section{Author Contributions}

Conceived and designed the experiments: HB CL JM LB MB MP. Performed the experiments: HB CL JM LB MB MP. Analyzed the data: HB CL JM LB MB MP. Wrote the paper: HB CL JM MB MP.

Diffley JF, Stillman B (1991) A close relative of the nuclear, chromosomal highmobility group protein HMG1 in yeast mitochondria. Proc Natl Acad Sci U S A 88: 7864-7868.

6. Bonawitz ND, Clayton DA, Shadel GS (2006) Initiation and beyond: multiple functions of the human mitochondrial transcription machinery. Mol Cell 24: 813-825.

7. Stigter D (2004) Packaging of single DNA molecules by the yeast mitochondrial protein Abf2p: reinterpretation of recent single molecule experiments. Biophys Chem 110: 171-178.

8. Friddle RW, Klare JE, Martin SS, Corzett M, Balhorn R, et al. (2004) Mechanism of DNA compaction by yeast mitochondrial protein Abf2p. Biophys J 86: 1632-1639. 
9. Zelenaya-Troitskaya O, Newman SM, Okamoto K, Perlman PS, Butow RA (1998) Functions of the high mobility group protein, Abf2p, in mitochondrial DNA segregation, recombination and copy number in Saccharomyces cerevisiae. Genetics 148: 1763-1776.

10. Dutnall RN, Pillus L (2001) Deciphering NAD-dependent deacetylases. Cell 105: 161-164.

11. Blander G, Guarente L (2004) The Sir2 family of protein deacetylases. Annu Rev Biochem 73: 417-435.

12. Rusche LN, Kirchmaier AL, Rine J (2003) The establishment, inheritance, and function of silenced chromatin in Saccharomyces cerevisiae. Annu Rev Biochem 72: 481-516.

13. Li C, Mueller JE, Bryk M (2006) Sir2 represses endogenous polymerase II transcription units in the ribosomal DNA nontranscribed spacer. Mol Biol Cell 17: 3848-3859.

14. Bryk M, Banerjee M, Murphy M, Knudsen KE, Garfinkel DJ, et al. (1997) Transcriptional silencing of Tyl elements in the RDN1 locus of yeast. Genes Dev 11: 255-269.

15. Smith JS, Boeke JD (1997) An unusual form of transcriptional silencing in yeast ribosomal DNA. Genes Dev 11: 241-254.

16. Pasero P, Bensimon A, Schwob E (2002) Single-molecule analysis reveals clustering and epigenetic regulation of replication origins at the yeast rDNA locus. Genes Dev 16: 2479-2484.

17. Pappas DL Jr, Frisch R, Weinreich M (2004) The NAD(+)-dependent Sir2p histone deacetylase is a negative regulator of chromosomal DNA replication. Genes Dev 18: 769-781.

18. Weinreich M, Palacios DeBeer MA, Fox CA (2004) The activities of eukaryotic replication origins in chromatin. Biochim Biophys Acta 1677: 142-157.

19. Vogelauer M, Rubbi L, Lucas I, Brewer BJ, Grunstein M (2002) Histone acetylation regulates the time of replication origin firing. Mol Cell 10: 1223-1233.

20. O'Rourke TW, Doudican NA, Mackereth MD, Doetsch PW, Shadel GS (2002) Mitochondrial dysfunction due to oxidative mitochondrial DNA damage is reduced through cooperative actions of diverse proteins. Mol Cell Biol 22: 4086-4093.

21. Epstein CB, Waddle JA, Hale Wt, Dave V, Thornton J, et al. (2001) Genomewide responses to mitochondrial dysfunction. Mol Biol Cell 12: 297-308.

22. Chelstowska A, Butow RA (1995) RTG genes in yeast that function in communication between mitochondria and the nucleus are also required for expression of genes encoding peroxisomal proteins. J Biol Chem 270: 18141-18146.

23. Spencer F, Gerring SL, Connelly C, Hieter P (1990) Mitotic chromosome transmission fidelity mutants in Saccharomyces cerevisiae. Genetics 124: 237-249.

24. Kaiser C, Michaelis S, Mitchell A (1994) Methods in Yeast Genetics. Cold Spring Harbor: Cold Spring Harbor Laboratory Press.

25. Nash P, Tang X, Orlicky S, Chen O, Gertler FB, et al. (2001) Multisite phosphorylation of a CDK inhibitor sets a threshold for the onset of DNA replication. Nature 414: 514-521.
26. Murray DB, Beckmann M, Kitano H (2007) Regulation of yeast oscillatory dynamics. Proc Natl Acad Sci U S A 104: 2241-2246.

27. Tu BP, Kudlicki A, Rowicka M, McKnight SL (2005) Logic of the yeast metabolic cycle: temporal compartmentalization of cellular processes. Science 310: 1152-1158.

28. Klevecz RR, Bolen J, Forrest G, Murray DB (2004) A genomewide oscillation in transcription gates DNA replication and cell cycle. Proc Natl Acad Sci U S A 101: 1200-1205.

29. Anderson RM, Latorre-Esteves M, Neves AR, Lavu S, Medvedik O, et al. (2003) Yeast life-span extension by calorie restriction is independent of NAD fluctuation. Science 302: 2124-2126.

30. Huang J, Moazed D (2003) Association of the RENT complex with nontranscribed and coding regions of rDNA and a regional requirement for the replication fork block protein Fob1 in rDNA silencing. Genes Dev 17: 2162-2176.

31. Aggarwal BD, Calvi BR (2004) Chromatin regulates origin activity in Drosophila follicle cells. Nature 430: 372-376.

32. Poloumienko A, Dershowitz A, De J, Newlon CS (2001) Completion of replication map of Saccharomyces cerevisiae chromosome III. Mol Biol Cell 12: 3317-3327.

33. Longtine MS, McKenzie A, 3rd, Demarini DJ, Shah NG, Wach A, et al. (1998) Additional modules for versatile and economical PCR-based gene deletion and modification in Saccharomyces cerevisiae. Yeast 14: 953-961.

34. Brachmann CB, Davies A, Cost GJ, Caputo E, Li J, et al. (1998) Designer deletion strains derived from Saccharomyces cerevisiae S288C: a useful set of strains and plasmids for PCR-mediated gene disruption and other applications. Yeast 14: 115-132.

35. Ghaemmaghami S, Huh WK, Bower K, Howson RW, Belle A, et al. (2003) Global analysis of protein expression in yeast. Nature 425: 737-741.

36. Bogomolnaya LM, Pathak R, Guo J, Cham R, Aramayo R, et al. (2004) Hymlp affects cell cycle progression in Saccharomyces cerevisiae. Curr Genet 46: 183-192.

37. Guo J, Bryan BA, Polymenis M (2004) Nutrient-specific effects in the coordination of cell growth with cell division in continuous cultures of Saccharomyces cerevisiae. Arch Microbiol 182: 326-330.

38. Pathak R, Bogomolnaya LM, Guo J, Polymenis M (2004) Gid8p (Dcrlp) and Dcr2p function in a common pathway to promote START completion in Saccharomyces cerevisiae. Euk Cell 3: 1627-1638.

39. Cross FR, Archambault V, Miller M, Klovstad M (2002) Testing a mathematical model of the yeast cell cycle. Mol Biol Cell 13: 52-70.

40. Bryk M, Briggs SD, Strahl BD, Curcio MJ, Allis CD, et al. (2002) Evidence that Set1, a factor required for methylation of histone $\mathrm{H} 3$, regulates rDNA silencing in S. cerevisiae by a Sir2-independent mechanism. Curr Biol 12: 165-170.

41. Baganz F, Hayes A, Farquhar R, Butler PR, Gardner DC, et al. (1998 Quantitative analysis of yeast gene function using competition experiments in continuous culture. Yeast 14: 1417-1427. 